\title{
NAD(P)H:quinone oxidoreductase-1 overexpression predicts poor prognosis in small cell lung cancer
}

\author{
XUELIAN CUI $^{1 *}$, TIEFENG JIN $^{1 *}$, XIAOYAN WANG ${ }^{1}$, GUANG JIN $^{1}$, ZHUHU LI $^{1}$ and LIJUAN LIN ${ }^{1,2}$ \\ ${ }^{1}$ Department of Pathology, Yanbian University College of Medicine, Yanji, Jilin 133002; \\ ${ }^{2}$ Department of Medical Imaging, College of Medicine, Eastern Liaoning University, Dandong, Liaoning 118002, P.R. China
}

Received June 4, 2014; Accepted August 6, 2014

DOI: $10.3892 /$ or.2014.3494

\begin{abstract}
NAD(P)H:quinone oxidoreductase-1 (NQO1) is commonly elevated in various types of human cancers, including pancreatic, breast and thyroid cancer, as well as others. However, little is known concerning the status of NQO1 in small cell lung cancer (SCLC). To investigate the clinicopathological significance of NQO1 expression and evaluate its role as a potential prognostic marker in SCLC, protein and mRNA expression levels of NQO1 were determined in four fresh tissue samples of SCLC and paired adjacent non-cancerous tissues using western blotting and real-time qRT-PCR, respectively, and 115 cases of SCLC with strict follow-up were selected for immunohistochemical (IHC) staining of NQO1 protein. The correlation between NQO1 expression and the clinicopathological features of SCLC was evaluated using the Chi-square $\left(\chi^{2}\right)$ and Fisher's exact tests, survival rates were calculated using the Kaplan-Meier method, and univariate and multivariate analyses were performed using the Cox proportional hazards regression model. In regards to the results, levels of NQO1 protein and mRNA were significantly elevated in the four fresh tissue samples of SCLC compared with levels in the paired adjacent non-cancerous tissues, respectively. IHC analysis showed that the rate of strong positive NQO1 protein expression was significantly higher $(48.70 \%)$ in SCLC when compared with the rate in either adjacent non-cancerous or normal lung tissues (both $\mathrm{P}<0.001$ ). The rate of strong positive NQO1 protein expression was correlated with large tumor size $(\mathrm{P}=0.019)$, late pathologic stage $(\mathrm{P}=0.001)$ and the presence of lymph node metastasis $(\mathrm{P}=0.001)$. Moreover, high-level expression of NQO1 protein was significantly correlated with
\end{abstract}

Correspondence to: Dr Lijuan Lin or Dr Zhuhu Li, Department of Pathology, Yanbian University Medical College, 977 Gongyuan Road, Yanji, Jilin 133002, P.R. China

E-mail: linlijuan3066@163.com

E-mail: lizh@ybu.edu.cn

${ }^{*}$ Contributed equally

Key words: NAD(P)H:quinone oxidoreductase-1, small cell lung cancer, prognosis, survival analysis lower disease-free survival $(\mathrm{P}=0.001)$ and 5-year survival rates $(\mathrm{P}<0.001)$ in SCLC patients, particularly early-stage patients $(\mathrm{P}=0.045$ and $\mathrm{P}=0.033$, respectively). Further Cox analysis revealed that NQO1 expression emerged as a significantly independent hazard factor for the 5-year survival rate of patients with SCLC $(\mathrm{P}=0.042)$. In conclusion, NQO1 plays an important role in the progression of SCLC, and it may potentially be used as a biomarker and therapeutic target of SCLC.

\section{Introduction}

Small cell lung cancer (SCLC), which represents $12.95 \%$ of all lung cancer diagnoses and continues to be a major clinical issue worldwide (1), is an aggressive neuroendocrine subtype of lung cancer for which there is no effective treatment (2). Patients with limited disease have a median survival time of 16-24 months when treated with chemotherapy and concurrent thoracic radiation (3). The mechanism of carcinogenesis, similar to other cancers, is still not fully understood. Therefore, there is an urgent need for a better understanding of SCLC pathogenesis that may lead to more effective treatment strategies.

NAD(P)H:quinone oxidoreductase-1 (NQO1), which was discovered by Professor Ernster in 1958 (4) and originally called DT-diaphorase (5), is located on chromosome 16q22 (6). Several functions of NQO1 have been proposed, including xenobiotic detoxification, superoxide scavenging, maintenance of endogenous antioxidants, modulation of p53 and proteasomal degradation (7). It is conceivable that NQO1 functions primarily to protect normal cells from oxidant stress and electrophilic attack. These functions have also led to the suggestion that NQO1 plays an important role in cancer chemoprevention. On the other hand, NQO1 is expressed at high levels in most human solid tumors including those of the colon, breast, pancreas, ovaries and thyroid (8-10), and has also been detected following the induction of cell cycle progression and proliferation of melanoma cells (11). However, to date, the expression status of NQO1 in SCLC and its relationship with clinicopathological features/prognosis is unknown.

The critical role of NQO1 in numerous cancers compelled us to study the function of NQO1 in SCLC. Therefore, we performed western blotting, real-time RT-PCR (qRT-PCR) and immunohistochemical (IHC) staining of NQO1 in SCLC and normal lung tissues and found that NQO1 may be an independent prognostic biomarker for SCLC. 


\section{Materials and methods}

Ethics statement. The present study complied with the Helsinki Declaration and was approved by the Human Ethics and Research Ethics Committees of Yanbian University Medical College in China. Through surgery consent form, patients were informed that the resected specimens would be stored by the hospital and would potentially be used for scientific research, and that their privacy would be maintained. Follow-up survival data were collected retrospectively through analysis of medical records.

Clinical samples. Fresh samples from four cases of SCLC were paired with adjacent non-cancerous tissues, and 115 cases of routinely processed and paraffin-embedded SCLC specimens with strict follow-up for survival status were selected randomly from patients who underwent surgery between 2003 and 2011 at Jilin University Hospital and the Tumor Tissue Bank of Yanbian University Medical College. The pathological parameters, including age, gender, smoking status, tumor size, location subtype, clinical stage, lymph node metastasis, neuron-specific enolase (NSE) level, disease-free and 5-year survival data, were carefully reviewed for all the above cases. The patient ages ranged from 26-76 years with a mean age of 49.6 years. The male to female ratio was 69:46. Tumors were staged according to the 7 th edition of the American Joint Committee on Cancer. Of the 115 SCLCs, 70 were early-stage (I-II), and 45 cases were late-stage (III). Forty-four cases were localized in the central bronchus, and 71 cases in the peripheral bronchus. There were no patients with distant metastasis prior to surgery. By March 2013, 99 patients had died while 16 patients remained alive. The median survival time was 27.2 months.

Western blotting. As previously described (12), fresh tissue samples of SCLC were ground into powder in liquid nitrogen and lysed in SDS-PAGE sample buffer. Equal amounts of protein samples $(20 \mu \mathrm{g})$ were separated on $12 \%$ SDS-polyacrylamide gels and transferred to PVDF membranes. Membranes were blocked with 5\% fat-free milk in Tris-buffered saline containing $0.1 \%$ Tween-20 for $1 \mathrm{~h}$ at room temperature (RT). Membranes were incubated with the NQO1 antibody (1:5,000; Santa Cruz Biotechnology, Santa Cruz, CA, USA) overnight at $4^{\circ} \mathrm{C}$ and then with horseradish peroxidase-conjugated rabbit anti-mouse IgG. NQO1 expression was detected using the ECL Prime Western Blotting detection reagent (Amersham) according to the manufacturer's instructions. $\beta$-actin (Sigma, St. Louis, MO, USA) was used as a loading control. Protein bands were quantified using the LANE 1D system (Sage, China).

$R N A$ extraction and $q R T-P C R$. Total RNA from fresh tissues was extracted using TRIzol reagent (Invitrogen, Carlsbad, CA, USA). First-strand cDNA was synthesized using PrimeScript reverse transcriptase (Takara Biotechnology, Dalian, China) and oligo(dT), following the manufacturer's instructions. To examine expression, qPCR was performed with a Bio-Rad sequence detection system according to the manufacturer's instructions using a double-stranded DNA-specific SYBR Premix Ex Taq ${ }^{\mathrm{TM}}$ II kit (Takara Biotechnology). Double-
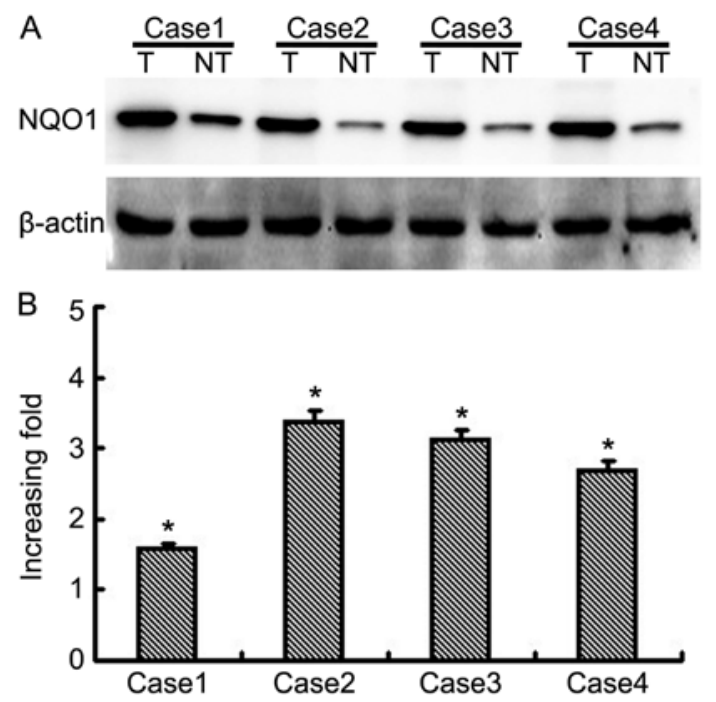

Figure 1. Western blot analysis of the NQO1 protein. (A) Images of western blot analyses of NQO1 protein expression in four matched pairs of SCLC (T) and adjacent non-tumor tissues (NT). (B) Relative T/NT ratios of NQO1 protein expression level in paired SCLC and adjacent non-tumor tissues (increased fold-change, $\left.{ }^{*} \mathrm{P}<0.05\right)$. NQO1, NAD $(\mathrm{P}) \mathrm{H}$ :quinone oxidoreductase-1; SCLC, small cell lung cancer.

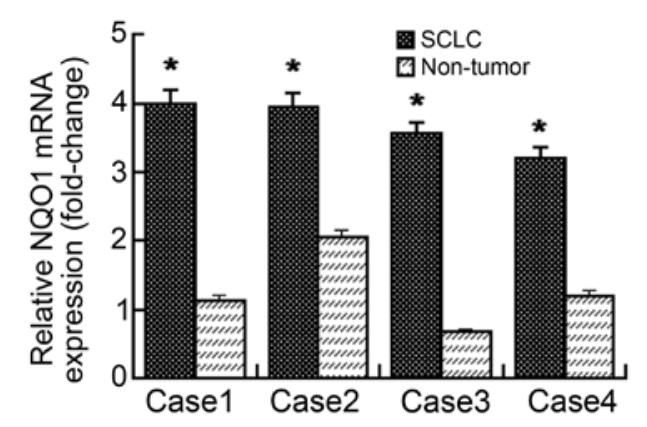

Figure 2. qRT-PCR analysis of NQO1 mRNA. SCLC and adjacent non-tumor tissues (non-tumor) were collected and subjected to qRT-PCR analysis of NQO1 mRNA levels. Data represent the mean of individual samples tested in triplicate relative to that of the normal control $\pm \mathrm{SD}\left({ }^{*} \mathrm{P}<0.05\right)$. NQO1, $\mathrm{NAD}(\mathrm{P}) \mathrm{H}$ :quinone oxidoreductase-1; SCLC, small cell lung cancer.

stranded DNA-specific expression was tested using the comparative $\mathrm{Ct}$ method using $2^{-\Delta \Delta \mathrm{Ct}}(13)$. NQO1 primers were: 5'-GGCAGAAGAGCACTGATCGTA-3' and 5'-TGATGGGA TTGAAGTTCATGGC-3'. Primers for GAPDH, which was used as an internal control, were: 5'-GGTCTCCTCTGACTTC AACA-3' and 5'-ATACCAGGAAATGAGCTTGA-3'. All assays were performed in triplicate and repeated at least three times.

IHC analysis. IHC analysis was performed using the Dako LSAB kit (Dako A/S, Glostrup, Denmark). Briefly, to eliminate endogenous peroxidase activity, $4-\mu \mathrm{m}$ tissue sections were deparaffinized, rehydrated and incubated with $3 \% \mathrm{H}_{2} \mathrm{O}_{2}$ in methanol for $15 \mathrm{~min}$ at RT. Antigen retrieval was performed at $95^{\circ} \mathrm{C}$ for $20 \mathrm{~min}$ by placing the slides in $0.01 \mathrm{M}$ sodium citrate buffer $(\mathrm{pH} 6.0)$. The slides were then incubated with the NQO1 antibody (1:500; Santa Cruz) at $4^{\circ} \mathrm{C}$ overnight. After incubation with the biotinylated secondary antibody at RT for $30 \mathrm{~min}$, the slides were incubated with streptavidin-peroxidase 
A
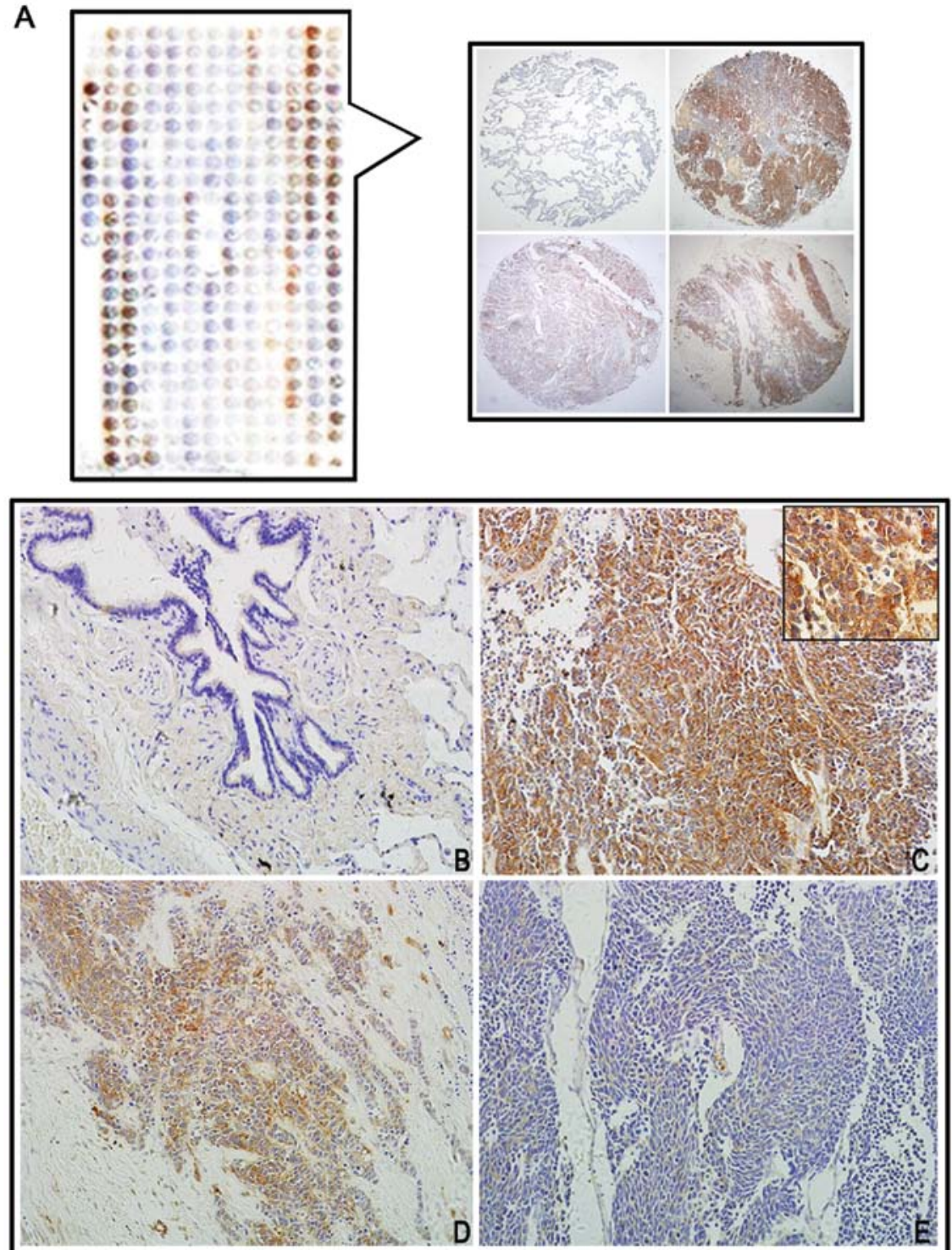

Figure 3. NQO1 protein expression in SCLC using IHC. (A) NQO1 protein expression in an SCLC tissue microarray. (B) NQO1 protein staining was negative in adjacent normal lung tissues. (C) NQO1 protein showed diffusely and strongly cytoplasmic (insert) positive staining in late-stage SCLC. (D) NQO1 protein staining was weakly positive in SCLC without metastasis. (E) NQO1 protein staining was negative in SCLC without metastasis. NQO1, NAD(P)H:quinone oxidoreductase-1; SCLC, small cell lung cancer; IHC, immunohistochemistry.

complex at RT for 30 min. Immunostaining was developed using 3,3'-diaminobenzidine, and Mayer's hematoxylin was used for counterstaining (14). We used mouse $\operatorname{IgG}$ as an isotope controls. In addition, positive tissue sections were processed while omitting the primary antibody (mouse anti-NQO1) as negative controls.

Two pathologists (T. Jin and Z. Li), blinded to all clinical data, independently scored all slides. Interpretation criteria were as previously described (13). Both cytoplasmic and nuclear NQO1 expression patterns were considered positive staining, and ++ or +++ scored samples were considered strongly positive. For survival data analysis, ++ or +++ scored samples were considered high and - or + scored samples were considered low NQO1 expression.

Statistical analysis. Statistical analyses were performed using SPSS 17.0. Correlation between NQO1 expression and clinicopathological characteristics was evaluated using the $\chi^{2}$ and Fisher's exact tests. Disease-free and 5-year survival rates after tumor removal were calculated by the Kaplan-Meier method, and difference in survival curves was analyzed by the log-rank test. Multivariate analysis was performed using the Cox proportional hazards regression model on all significant characteristics determined using univariate analysis. A P-value of $<0.05$ was considered to indicate a statistically significant result.

\section{Results}

NQO1 protein and $m R N A$ expression in SCLC. Expression levels of NQO1 protein and mRNA were determined in four SCLC samples with matched adjacent non-cancerous fresh tissues. Western blotting data showed that the NQO1 protein was highly expressed in SCLC tissues compared with the 
Table I. NQO1 protein expression in the SCLC cases.

\begin{tabular}{|c|c|c|c|c|c|c|c|}
\hline \multirow[b]{2}{*}{ Diagnosis } & \multirow{2}{*}{$\begin{array}{l}\text { No. of } \\
\text { cases }\end{array}$} & \multicolumn{4}{|c|}{ NQO1 protein expression } & \multirow{2}{*}{$\begin{array}{l}\text { Positive } \\
\text { rate (\%) }\end{array}$} & \multirow{2}{*}{$\begin{array}{l}\text { Strongly positive } \\
\text { rate }(\%)\end{array}$} \\
\hline & & - & + & ++ & +++ & & \\
\hline SCLC & 115 & 34 & 25 & 23 & 33 & $70.43^{\mathrm{a}}$ & $48.70^{\mathrm{a}}$ \\
\hline Adjacent non-tumor & 115 & 85 & 18 & 7 & 5 & 26.09 & 10.43 \\
\hline Normal lung tissues & 14 & 11 & 2 & 1 & 0 & 21.43 & 7.14 \\
\hline
\end{tabular}

Statistical analyses were performed using Pearson's Chi-Square test. ${ }^{\mathrm{P}} \mathrm{P}<0.01$ compared with either adjacent non-tumor or normal lung tissues. NQO1, NAD(P)H:quinone oxidoreductase-1; SCLC, small cell lung cancer.

level in the matched adjacent non-cancerous tissues (Fig. 1). qRT-PCR data also confirmed the increased level of NQO1 mRNA expression in the SCLC samples when compared with the level in the adjacent non-cancerous tissues (Fig. 2).

NQO1 protein expression showed a mainly cytoplasmic staining pattern in SCLC using IHC. The positive and strongly positive rates of NQO1 protein expression were $70.43 \%$ and $48.70 \%$, respectively, which was significantly higher than the rates in either adjacent non-cancerous or normal lung tissues $(\mathrm{P}<0.001$, respectively) (Fig. 3, Table I).

Correlation between NQO1 expression and clinicopathological features of SCLC. The correlation between NQO1 protein expression and the major clinicopathological features of SCLC was analyzed. The results showed that the NQO1 high-expression rate was significantly correlated with large tumor size $(\mathrm{P}=0.019)$, late pathologic stage $(\mathrm{P}=0.001)$ and lymph node metastasis $(\mathrm{P}=0.001)$. However, NQO1 expression levels did not correlate with the pathological parameters of SCLC cases including age, gender, smoking status, location subtype or NSE level ( $\mathrm{P}>0.05$, respectively) (Table II).

NQO1 high-level expression predicts poor survival rates in patients with SCLC using the Kaplan-Meier method. To further confirm the role of NQO1 expression in SCLC progression, we analyzed disease-free survival and 5-year survival rates for 115 SCLC cases using the Kaplan-Meier method, and found that SCLC patients with NQO1 high-level expression had lower disease-free $(\log$-rank=11.938, $\mathrm{P}=0.001)$ and 5-year survival rates $(\log -r a n k=12.899, \mathrm{P}<0.001)$ than those with NQO1 low-level expression (Fig. 4).

To further substantiate the importance of NQO1 expression in SCLC progression, we analyzed the correlation between NQO1 high-level expression rate and clinical stages of SCLC. In early-stage SCLC, patients with NQO1 high-level expression had lower disease-free and 5-year survival rates compared with those with NQO1 low-level expression ( $\mathrm{P}=0.045$ and $\mathrm{P}=0.033$, respectively) (Fig. 5A and B). However, in late-stage SCLC patients, disease-free and 5-year survival rates were not correlated with the NQO1 expression status $(\mathrm{P}=0.085$ and $\mathrm{P}=0.104$, respectively) (Fig. 5C and D).

NQO1 is an independent prognostic factor in SCLC using the Cox proportional hazards regression model. Upon univariate analysis, patients whose SCLC tumors with NQO1
Table II. Correlation between NQO1 expression and clinicopathological features of the SCLC cases.

\begin{tabular}{|c|c|c|c|c|}
\hline \multirow[b]{2}{*}{ Variables } & \multicolumn{2}{|c|}{ NQO1-positive cases (\%) } & \multirow[b]{2}{*}{$\chi^{2}$} & \multirow[b]{2}{*}{ P-value } \\
\hline & $+++/++$ & $+/-$ & & \\
\hline \multicolumn{5}{|l|}{ Age } \\
\hline$\leq 50$ & $27(47.37)$ & $30(52.63)$ & 0.080 & 0.778 \\
\hline$>50$ & $29(50.00)$ & $29(50.00)$ & & \\
\hline \multicolumn{5}{|l|}{ Gender } \\
\hline Male & $35(50.72)$ & $34(49.28)$ & 0.284 & 0.594 \\
\hline Female & $21(45.65)$ & $25(54.35)$ & & \\
\hline \multicolumn{5}{|c|}{ Smoking status } \\
\hline Yes & $33(45.83)$ & $39(54.17)$ & 0.631 & 0.427 \\
\hline No & $23(53.49)$ & $20(46.51)$ & & \\
\hline \multicolumn{5}{|l|}{ Tumor size } \\
\hline$\leq 3 \mathrm{~cm}$ & $30(40.54)$ & $44(59.46)$ & 5.525 & $0.019^{\mathrm{a}}$ \\
\hline$>3 \mathrm{~cm}$ & $26(63.41)$ & $15(36.59)$ & & \\
\hline \multicolumn{5}{|c|}{ Location subtype } \\
\hline Central & $23(52.27)$ & $21(47.73)$ & 0.365 & 0.546 \\
\hline Peripheral & $33(46.48)$ & $38(53.32)$ & & \\
\hline \multicolumn{5}{|l|}{ Clinical stage } \\
\hline I-II & $25(35.71)$ & $45(64.29)$ & 12.066 & $0.001^{\mathrm{b}}$ \\
\hline III & $31(68.89)$ & $14(31.11)$ & & \\
\hline \multicolumn{5}{|l|}{$\begin{array}{l}\text { Lymph node } \\
\text { metastasis }\end{array}$} \\
\hline Positive & $37(63.79)$ & $21(36.21)$ & 10.676 & $0.001^{\mathrm{b}}$ \\
\hline Negative & $19(33.33)$ & $38(66.67)$ & & \\
\hline \multicolumn{5}{|l|}{ NSE level } \\
\hline$\geq 16.3 \mathrm{ng} / \mathrm{ml}$ & $37(53.62)$ & $32(46.38)$ & 1.677 & 0.195 \\
\hline$<16.3 \mathrm{ng} / \mathrm{ml}$ & $19(41.30)$ & 27 (58.70) & & \\
\hline
\end{tabular}

Statistical analyses were performed using Pearson's Chi-square test. ${ }^{\mathrm{a}} \mathrm{P}<0.05, \quad{ }^{\mathrm{b}} \mathrm{P}<0.01$. NQO1, NAD $(\mathrm{P}) \mathrm{H}:$ quinone oxidoreductase-1; SCLC, small cell lung cancer; NSE, neuron-specific enolase.

high-level expression had a significantly lower 5-year survival rate $(\mathrm{P}=0.001)$ than those with NQO1 low-level expression 

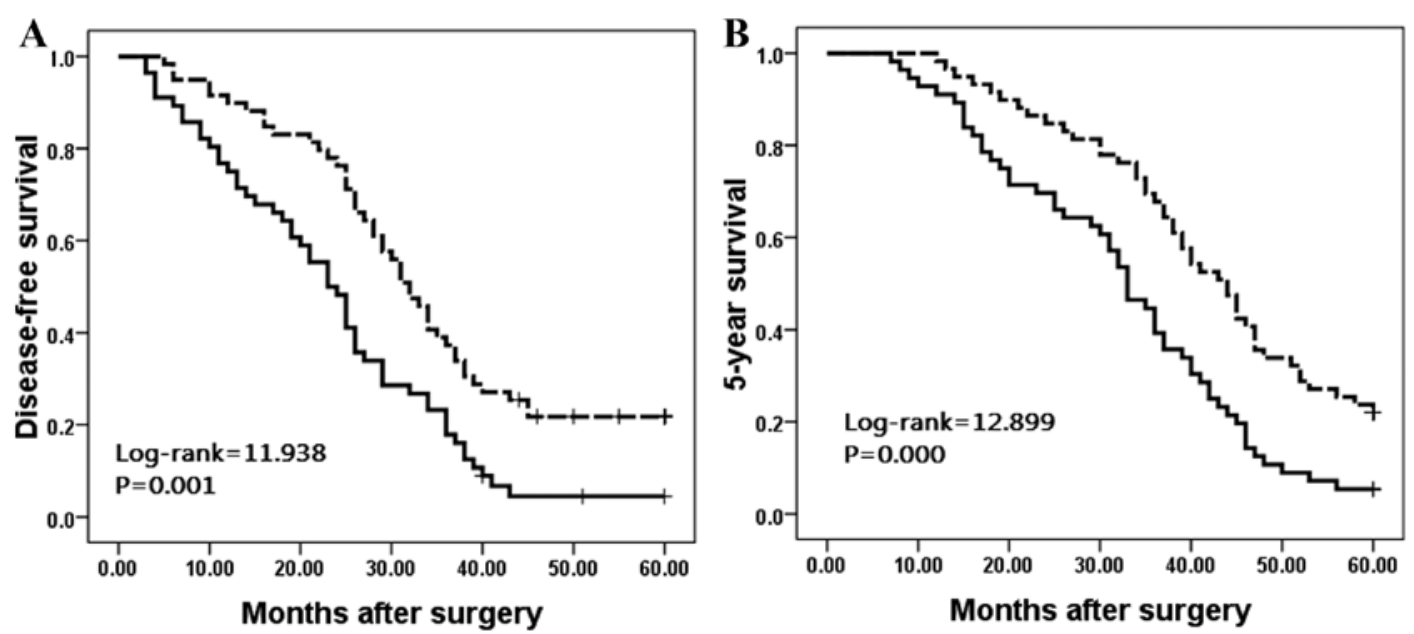

Figure 4. Kaplan-Meier analysis of SCLC patient survival rates in relation to NQO1 protein expression. Disease-free survival (A) and overall (5-year) survival rates (B) of patients with elevated (solid, $n=56$ ) and low (dashed, $n=59$ ) NQO1 expression. SCLC, small cell lung cancer; NQO1, NAD(P)H:quinone oxidoreductase-1.
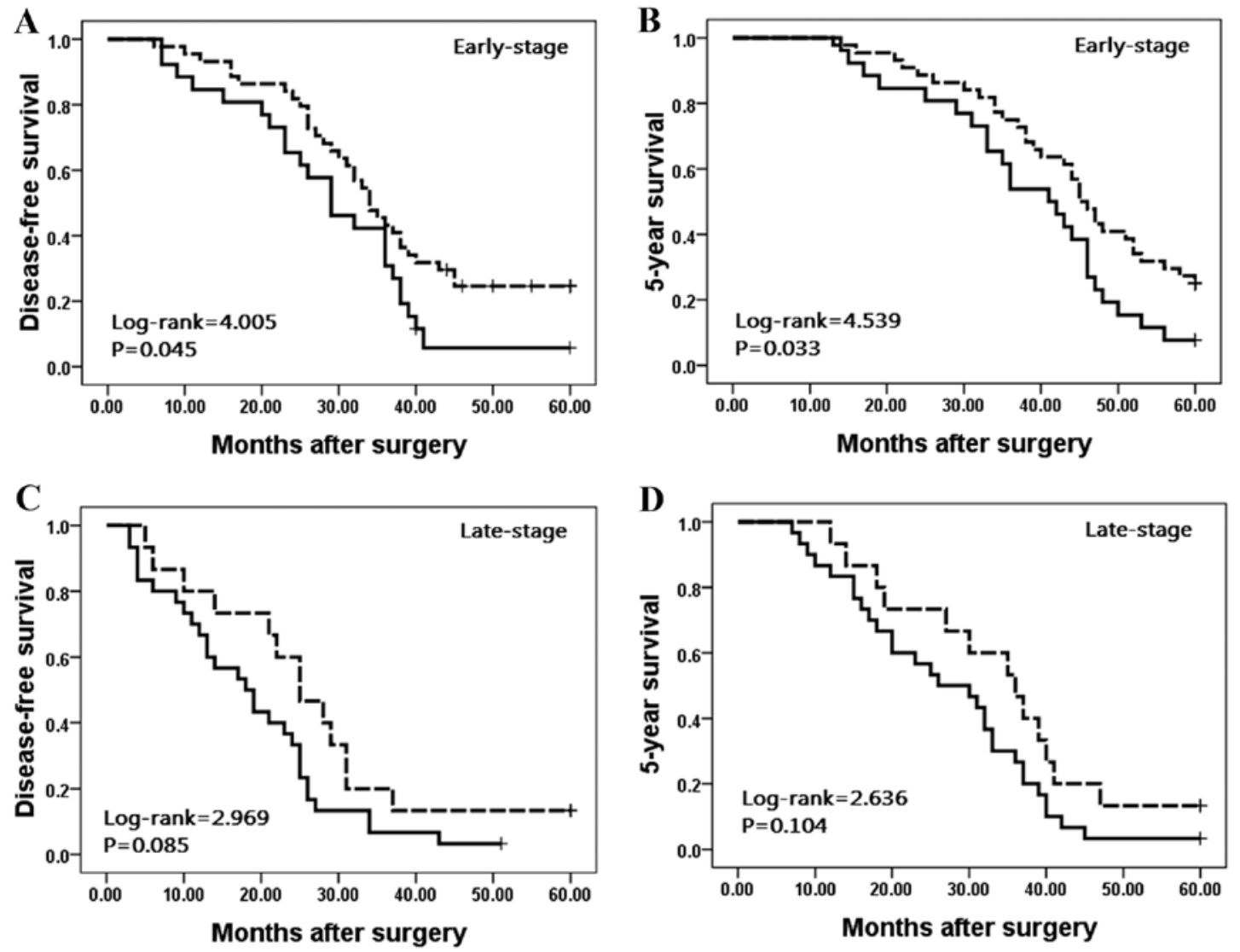

Figure 5. Kaplan-Meier analysis of the survival rates in patients with high- or low-level NQO1 expression with early- or late-stage SCLC. Disease-free survival (A) and overall (5-year) survival rates (B) were assessed in patients with early-stage SCLC concomitant with either high- (solid, $\mathrm{n}=25$ ) or low-level (dashed, $\mathrm{n}=45$ ) NQO1 expression. (C) Disease-free survival and (D) overall (5-year) survival rates were also assessed in patients with late-stage SCLC concomitant with high- (solid, $\mathrm{n}=31$ ) or low-level (dashed, $\mathrm{n}=14$ ) NQO1 expression. NQO1, NAD(P)H:quinone oxidoreductase-1; SCLC, small cell lung cancer.

tumors. Additionally, patient age $(\mathrm{P}=0.025)$, tumor location subtype $(\mathrm{P}=0.014)$, pathologic stage $(\mathrm{P}<0.001)$ and lymph node metastasis $(\mathrm{P}=0.044)$ were also associated with 5 -year survival rates. Therefore, multivariate survival analysis was then performed using the Cox proportional hazards regression model for all significant variables in the univariate survival analysis. We found that clinical stage (HR, 1.948; 95\% CI, 1.292-2.938, $\mathrm{P}=0.001$ ) proved to be an independent prognostic factor for 5-year survival rates in SCLC. More importantly, NQO1 expression also emerged as a significant independent prognostic factor in the prognosis of SCLC (HR, 1.577; 95\% CI, 1.017-2.446, $\mathrm{P}=0.042$ ) (Table III). 
Table III. Univariate and multivariate analysis of clinicopathological factors for the 5-year survival rate of 115 patients with SCLC.

\begin{tabular}{|c|c|c|c|c|c|c|c|}
\hline \multirow[b]{2}{*}{ Characteristics } & \multirow[b]{2}{*}{ B } & \multirow[b]{2}{*}{ SE } & \multirow[b]{2}{*}{ Wald } & \multirow[b]{2}{*}{$\mathrm{HR}$} & \multicolumn{2}{|c|}{$95 \%$ CI } & \multirow[b]{2}{*}{ P-value } \\
\hline & & & & & Lower & Upper & \\
\hline \multicolumn{8}{|c|}{ Univariate analysis } \\
\hline Age & 0.425 & 0.190 & 5.017 & 1.530 & 1.055 & 2.220 & $0.025^{\mathrm{a}}$ \\
\hline Gender & 0.078 & 0.192 & 0.167 & 1.081 & 0.743 & 1.574 & 0.683 \\
\hline Smoking status & 0.083 & 0.188 & 0.196 & 1.087 & 0.752 & 1.571 & 0.658 \\
\hline Tumor size & 0.354 & 0.194 & 3.349 & 1.425 & 0.975 & 2.082 & 0.067 \\
\hline Location & 0.477 & 0.195 & 5.978 & 1.611 & 1.099 & 2.360 & $0.014^{\mathrm{a}}$ \\
\hline Stage & 0.810 & 0.197 & 16.917 & 2.248 & 1.528 & 3.308 & $0.000^{\mathrm{b}}$ \\
\hline LN metastasis & 0.383 & 0.190 & 4.061 & 1.467 & 1.011 & 2.130 & $0.044^{\mathrm{a}}$ \\
\hline NSE level & 0.215 & 0.192 & 1.250 & 1.240 & 0.851 & 1.806 & 0.264 \\
\hline NQO1 & 0.643 & 0.192 & 11.229 & 1.902 & 1.306 & 2.771 & $0.001^{\mathrm{b}}$ \\
\hline \multicolumn{8}{|c|}{ Multivariate analysis } \\
\hline Age & 0.327 & 0.199 & 2.703 & 1.387 & 0.939 & 2.048 & 0.100 \\
\hline Location & 0.381 & 0.212 & 3.217 & 1.463 & 0.965 & 2.218 & 0.073 \\
\hline Stage & 0.667 & 0.210 & 10.119 & 1.948 & 1.292 & 2.938 & $0.001^{\mathrm{b}}$ \\
\hline LN metastasis & 0.268 & 0.220 & 1.490 & 1.307 & 0.850 & 2.011 & 0.222 \\
\hline NQO1 & 0.455 & 0.224 & 4.134 & 1.577 & 1.017 & 2.446 & $0.042^{\mathrm{a}}$ \\
\hline
\end{tabular}

Statistical analyses were performed using the Cox proportional hazards regression model. ${ }^{\mathrm{a} P}<0.05$, ${ }^{\mathrm{b}} \mathrm{P}<0.01$. SCLC, small cell lung cancer; NSE, neuron-specific enolase; NQO1, NAD(P)H:quinone oxidoreductase-1.

\section{Discussion}

NQO1 is a mainly cytosolic enzyme that uses NADH or NADPH as substrates to catalyze the two-electron reduction of quinines to their hydroquinone forms (15), thus bypassing the toxic semiquinone intermediates, and these resultant hydroquinones are thus ready for further conjugation and excretion (16). In addition, the enzyme is present as a ubiquitous flavoprotein that is widely expressed in body tissues (17) and localized primarily in the cytosol, although it has been detected in the nucleus at lower levels (18). NQO1 is induced along with a battery of defense genes that provide protection against different stresses as well as carcinogen-induced organ tumorigenesis.

The activity of the NQO1 enzyme can be influenced by a major polymorphism involving a single $\mathrm{C}$ to $\mathrm{T}$ substitution at nucleotide 609 in exon 6 of the NQO1 gene that causes a Pro187Ser amino acid change in the protein (19). Some studies have shown that the polymorphism in the NQO1 gene affects the translation of the NQO1 protein. The NQO1 C609T polymorphism has been associated with an increased risk of various cancers such as renal (20), lung (21), esophageal (22), colorectal (23), and head and neck cancer (24). Therefore, since there is an increased incidence of disease and xenobiotic-induced toxicity in individuals carrying a polymorphism in NQO1, it has been suggested that it plays a role in chemoprotection.

Recently, however, it was reported that NQO1 protein and mRNA were abnormally elevated in many solid tumors. Mikami et al (25) demonstrated a close correlation between protein expression and enzyme activity of NQO1 in colon cell lines and in colorectal tumor samples, and tumors with nodal metastases showed significantly higher NQO1 levels than did tumors without metastasis, raising the possibility that NQO1 expression is related to tumorigenesis and malignant progression of colorectal tumors.

Awadallah et al (10) and Lyn-Cook et al (8) reported that NQO1 is upregulated in pancreatic ductal adenocarcinoma (PDAC), and that combining NQO1 expression with cellular morphology assessment minimized the risk of a false-positive diagnosis. A study by Fagerholm et al (26) in breast cancer patients showed that NQO1 may predict the outcome of patients with overabundant expression of the Fanconi anemia group D2 (FANCD2) protein through its potential modification of prognostic DNA repair markers. A study by $\mathrm{Li}$ et al (27) highlighted that NQO1 was not only a 'biomarker', but also an exploitable and tumor-selective target for PDAC. However, little is known concerning the status of NQO1 in SCLC.

In the present study, qRT-PCR and western blotting data demonstrated that the levels of NQO1 mRNA and protein were significantly higher in SCLC samples compared with adjacent non-tumor tissues. Furthermore, we performed IHC staining and analysis of 115 cases of SCLC, and found that a strongly positive rate of NQO1 protein expression was significantly higher than that observed in either adjacent non-tumor or normal lung tissues, indicating that NQO1 plays a potentially important role in the progression of SCLC. Meanwhile, we analyzed the correlation between NQO1 expression and clinicopathological features of SCLC and found that NQO1 expression was significantly correlated with large tumor size, 
late pathologic stage and lymph node metastasis $(\mathrm{P}<0.05)$, but not with age, gender, smoking status, location subtype or NSE level $(\mathrm{P}>0.05)$.

With regard to survival, we found that SCLC patients with high-level NQO1 expression had lower disease-free survival $(\mathrm{P}<0.01)$ and 5 -year survival rates $(\mathrm{P}<0.01)$ than patients with low-level NQO1 expression. In early-stage SCLC, patients with high-level NQO1 expression had lower disease-free and overall survival rates compared with those with low NQO1 expression $(\mathrm{P}<0.05)$. Multivariate survival analysis demonstrated that NQO1 expression emerged as a significant independent hazard factor for overall (5-year) survival in SCLC along with clinical stage. These results demonstrate that NQO1 is a potential effective predictor of poor prognosis, particularly in patients with early-stage SCLC.

Collectively, the present study implicates NQO1 as a putative new attractive biomarker for prognostic evaluation and as a potential molecular target in patients with SCLC. Additionally, Vasiliou et al (28) indicated that NQO1 was shown to be upregulated as part of the oxidative stress response and inexplicably overexpressed in particular types of tumors, whose function has not yet been elucidated. Garate et al (11) attempted to determine the expression and basic function of NQO1 in melanoma cell proliferation, and found that NQO1 significantly induced cell cycle progression by upregulating the expression of cyclin A2, B1 and D1, leading to the proliferation of melanoma cells in vitro. In spite of this, the molecular mechanism of NQO1 affecting tumor progression is still not clearly understood. NQO1 may constitute an undiscovered signaling molecule in cancer cells, and further studies are warranted to explore this possibility.

\section{Acknowledgements}

This study was supported by grants from the National Natural Science Funds of China (61371067), and the International Cooperative Project from the Jilin Provincial Science and Technology Department in China (20140414050GH).

\section{References}

1. Kallianos A, Rapti A, Zarogoulidis P, et al: Therapeutic procedure in small cell lung cancer. J Thorac Dis 5 (Suppl 4): S420-S424, 2013.

2. Rudin CM, Hann CL, Peacock CD and Watkins DN: Novel systemic therapies for small cell lung cancer. J Natl Compr Cancer Netw 6: 315-322, 2008.

3. Zhang Y and He J: The development of targeted therapy in small cell lung cancer. J Thorac Dis 5: 538-548, 2013.

4. Siegel D and Ross D: Immunodetection of NAD $(\mathrm{P}) \mathrm{H}$ :quinine oxidoreductase 1 (NQO1) in human tissues. Free Radic Biol Med 29: 246-253, 2000.

5. Siegel D, Yan C and Ross D: NAD(P)H:quinone oxidoreductase 1 (NQO1) in the sensitivity and resistance to antitumor quinones. Biochem Pharmacol 83: 1033-1040, 2012.

6. Zhu CL, Huang Q, Liu CH, et al: $\mathrm{NAD}(\mathrm{P}) \mathrm{H}$ : quinone oxidoreductase 1 (NQO1) C609T gene polymorphism association with digestive tract cancer: a meta-analysis. Asian Pac J Cancer Prev 14: 2349-2354, 2013.

7. Buranrat B, Prawan A, Kukongviriyapan U, et al: Dicoumarol enhances gemcitabine-induced cytotoxicity in high NQO1expressing cholangiocarcinoma cells. World J Gastroenterol 16 2362-2370, 2010.
8. Lyn-Cook BD, Yan-Sanders Y, Moore S, et al: Increased levels of $\mathrm{NAD}(\mathrm{P}) \mathrm{H}$ : quinine oxidoreductase 1 (NQO1) in pancreatic tissues from smokers and pancreatic adenocarcinomas: a potential biomarker of early damage in the pancreas. Cell Biol Toxicol 22: 73-80, 2006.

9. Cheng Y, Li J, Martinka M and Li G: The expression of NAD(P)H:quinone oxidoreductase 1 is increased along with $\mathrm{NF}-\kappa \mathrm{B}$ p105/p50 in human cutaneous melanomas. Oncol Rep 23: 973-979, 2010.

10. Awadallah NS, Dehn D, Shah RJ, et al: NQO1 expression in pancreatic cancer and its potential use as a biomarker. Appl Immunohistochem Mol Morphol 16: 24-31, 2008.

11. Garate M, Wani AA and Li G: The NAD(P)H:quinone oxidoreductase 1 induces cell cycle progression and proliferation of melanoma cells. Free Radic Biol Med 48: 1601-1609, 2010.

12. Liu S, Li L, Zhang Y, et al: The oncoprotein HBXIP uses two pathways to up-regulate S100A4 in promotion of growth and migration of breast cancer cells. J Biol Chem 287: 30228-30239, 2012.

13. Kong J, Li Y, Liu S, et al: High expression of ezrin predicts poor prognosis in uterine cervical cancer. BMC Cancer 13: 520, 2013.

14. Lin L, Piao J, Gao W, et al: DEK over expression as an independent biomarker for poor prognosis in colorectal cancer. BMC Cancer 13: 366, 2013.

15. Lajin B and Alachkar A: The NQO1 polymorphism C609T (Pro187Ser) and cancer susceptibility: a comprehensive metaanalysis. Br J Cancer 109: 1325-1337, 2013.

16. Talalay $\mathrm{P}$ and Dinkova-Kostova AT: Role of nicotinamide quinine oxidoreductase 1 (NQO1) in protection against toxicity of electrophiles and reactive oxygen intermediates. Methods Enzymol 382: 355-364, 2004.

17. Lee J, Kim KS, Lee MH, et al: $\mathrm{NAD}(\mathrm{P}) \mathrm{H}$ : quinone oxidoreductase 1 and NRH:quinone oxidoreductase 2 polymorphisms in papillary thyroid microcarcinoma: correlation with phenotype. Yonsei Med J 54: 1158-1167, 2013.

18. Nair S, Xu C, Shen G, et al: Pharmacogenomics of phenolic antioxidant butylated hydroxyanisole (BHA) in the small intestine and liver of Nrf2 knockout and C57L/6J mice. Pharm Res 23: 2621-2637, 2006.

19. Kuehl BL, Paterson JW, Peacock JW, et al: Presence of a heterozygous substitution and its relationship to DT-diaphorase activity. Br J Cancer 72: 555-561, 1995.

20. Schulz WA, Krummeck A, Rösinger I, et al: Increased frequency of a null-allele for $\mathrm{NAD}(\mathrm{P}) \mathrm{H}$ : quinone oxidoreductase in patients with urological malignancies. Pharmacogenetics 7: 235-239, 1997.

21. Nagata M, Kimura T, Suzumura T, et al: C609T polymorphism of NADPH quinone oxidoreductase 1 correlates clinical hematological toxicities in lung cancer patients treated with amrubicin. Clin Med Insights Oncol 7: 31-39, 2013.

22. Malik MA, Zargar SA and Mittal B: Role of NQO1 609C>T and $\mathrm{NQO} 2-3423 \mathrm{G}>\mathrm{A}$ gene polymorphisms in esophageal cancer risk in Kashmir valley and meta analysis. Mol Biol Rep 39: 9095-9104, 2012

23. Singh M, Goel S, Meeley RB, et al: Production of viable gametes without meiosis in maize deficient for an ARGONAUTE protein. Plant Cell 23: 443-458, 2011.

24. Soucek P, Susova S, Mohelnikova-Duchonova B, et al: Polymorphisms in metabolizing enzymes and the risk of head and neck squamous cell carcinoma in the Slavic population of the central Europe. Neoplasma 57: 415-421, 2010.

25. Mikami K, Naito M, Ishiguro T, et al: Immunological quantitation of DT-diaphorase in carcinoma cell lines and clinical colon cancers: advanced tumors express greater levels of DT-diaphorase. Jpn J Cancer Res 89: 910-915, 1998.

26. Fagerholm R, Sprott K, Heikkinen T, et al: Overabundant FANCD2, alone and combined with NQO1, is a sensitive marker of adverse prognosis in breast cancer. Ann Oncol 24: 2780-2785, 2013.

27. Li LS, Bey EA, Dong Y, et al: Modulating endogenous NQO1 levels identifies key regulatory mechanisms of action of $\beta$-lapachone for pancreatic cancer therapy. Clin Cancer Res 17: 275-285, 2011.

28. Vasiliou V, Ross D and Nebert DW: Update of the NAD(P) H:quinine oxidoreductase $(N Q O)$ gene family. Hum Genomics 2: 329-335, 2006. 